\title{
Congenital muscular dystrophy with severe retrocollis and mental retardation: a report of two siblings
}

\author{
Lina Nashef, B D Lake, A H V Schapira
}

\begin{abstract}
Two siblings with a congenital muscular dystrophy and severe mental retardation which was not due to dystrophin, merosin, or adhalin deficiency are described. These cases overlap with congenital muscular dystrophy of the Fukuyama-type but are less severe. Atypical features include limited facial involvement, retained ambulation, and severe retrocollis.
\end{abstract}

$(\mathcal{F}$ Neurol Neurosurg Psychiatry 1997;62:279-281)

Keywords: congenital muscular dystrophy; retrocollis; mental retardation; congenital muscular dystrophy of Fukuyama-type

The congenital muscular dystrophies are a heterogeneous group of disorders. A classification comprising four clinical phenotypes is listed by Dubowitz. ${ }^{1}$ These consist of "pure" congenital muscular dystrophy (the classic or occidental form), Fukuyama-type congenital muscular dystrophy, muscle-eye-brain disease (Santavouri), and Walker-Warburg syndrome. These clinical phenotypes may not all represent independent genetic entities. Furthermore, this classification does not take into account the results of immunocytochemical staining of muscle.

Fukuyama-type congenital muscular dystrophy is the second most common muscular dystrophy in Japan and is characterised by onset before the age of 1 year, severe motor impairment with prominent early contractures, and involvement of the CNS. ${ }^{12}$ Most cases survive beyond childhood into adolescence. ${ }^{3}$ The Fukuyama-type congenital muscular dystrophy locus has been mapped to $9 q 31-33^{4}$ but the gene has not yet been identified. The range of the clinical phenotype remains unknown with debate regarding the inclusion of the more severe but overlapping clinical syndromes of muscle-eye-brain disease and Walker-Warburg syndrome. ${ }^{35}$ Recent genetic analysis of a sibship with both Fukuyama-type congenital muscular dystrophy and Walker-Warburg syndrome suggests that these phenotypes could be genetically identical. ${ }^{26}$ In muscle-eye-brain disease but not Fukuyama-type congenital muscular dys- trophy, on the other hand, progressive myopia, glaucoma, and late cataracts are typical. A recent linkage study in seven Finnish families with muscle-eye-brain disease excluded the region harbouring Fukuyama-type congenital muscular dystrophy, the authors concluding that the two diseases are not allelic. ${ }^{7}$ Finally, abnormalities in dystrophin staining have been reported occasionally in Fukuyama-type congenital muscular dystrophy. ${ }^{8}$ Both a combined genotype and secondary deficiency have been offered as explanations for this finding.

We describe the occurrence of a congenital muscular dystrophy and severe mental retardation in two siblings of Asian extraction which was not due to dystrophin, merosin, or adhalin deficiency. Our cases overlap with, but are not typical of, Fukuyama-type congenital muscular dystrophy, having less severe motor involvement and severe retrocollis.

\section{Case reports}

CASE 1

The patient, a 21 year old man, was born to unrelated Pakistani parents. One sister was similarly affected and three other siblings are normal. Pregnancy was uncomplicated with normal intrauterine movements and birth. No neonatal difficulties were noted but milestones were delayed. He crawled at the age of 2, walked at the age of 3.5 years, but was never able to run. Gowers' manoeuvre was seen early on. His best age for walking was reported to be 7-8 years, after which his gait deteriorated gradually although he is still able to walk a few steps unaided. He developed retrocollis at about the age of 14 . He has always been mentally retarded with no evidence of regression other than some recent occasional episodes of nocturnal incontinence. He spoke his first words at the age of 2.5 years. Speech development was poor and his speech is currently limited to some 15 words. He swallows soft foods easily but can choke on large pieces. At the age of 7 years one probable seizure was witnessed when he suddenly became blank, stiff, and collapsed. No treatment was given and no further episodes occurred.

On examination, he had severe retrocollis which was maintained during sleep. Active contraction of neck extensors was present and confirmed by EMG. He walked with his neck 
fully extended and his face upwards, and when seated he was unable to face forward unless fully flexed at the waist, a frog-like position of his trunk which he always adopted. He was alert, conscious of his environment, and had some social interaction but was severely mentally retarded. He used single words and these only sparingly. Facial involvement was mild and eye movements were full. He had a prominent protruding lower lip. Contractures of the elbows and knees were slight. No pseudohypertrophy of any muscle groups was noted and there was no clinical evidence of bulbar or cardiac involvement. Formal grading of power was not possible, but he had clear proximal limb and girdle wasting with pronounced weakness and depressed reflexes.

Creatine kinase concentration was 5418 (normal range 20-200) IU/1. EMG was limited to paraspinal muscles (splenius capitus and semispinalis) and showed continuous muscle activity. Muscle biopsy was performed on two occasions (at ages 7 and 21 years). The first was reported as showing pronounced variation in fibre diameter, increased numbers of central nuclei, and increased endomysial connective tissue. Some foci of fibre necrosis and occasional regenerating fibres were seen with no inflammatory changes. The second biopsy from the right quadriceps showed islands of muscle fibres embedded in connective tissue. The muscle fibres that were present showed large variations in diameter with internal nuclei commonly seen but no necrosis or phagocytosis. The appearances indicated a longstanding dystrophic process. Immunostaining for merosin, adhalin, and DAG-43 (dystrophin associated glycoprotein) was normal. Dystrophin I and III staining was normal. Dystrophin II staining showed a few fibres with patchy negative areas. EEG showed some excess theta activity and paucity of background rhythm but no focal or epileptiform features. Cervical spine MRI was within normal limits. High resolution volumetric MRI of the brain ( 1.5 Tesla) was performed with three dimensional reconstructions and surface rendering of gyral patterns. This showed enlarged ventricles and prominent sulci but no migrational abnormalities or abnormalities of cortical gyrae. An ECG showed deep $S$ waves in limb leads and a prominent $R 1$ in left sided chest leads.

CASE 2

An older sister of patient 1 had been similarly but less severely affected although she had prominent contractures and a severe scoliosis. Again pregnancy, delivery, and neonatal period were normal. She sat up at 10 months and walked at the age of 4 . She was able to run but never normally. She was mentally retarded and had had one probable seizure. At the age of 15 a scoliosis was noted and she began to develop cervical hyperextension. When examined at the age of 18 , she was noted to have hyperextension of the cervical spine, a thoracolumbar scoliosis, fixed flexion deformities of the hips and knees, and contractures of the elbows. To achieve forward vision she sat, like her brother, with her trunk on her thighs. She was retarded and although she spoke she often repeated the same words. Creatine kinase concentration was 1140 (normal < 130) IU/1. Muscle biopsy was not performed. EMG was myopathic with no spontaneous activity. An EEG showed a moderate excess of slow components and CT of the brain showed slight enlargement of the lateral ventricles and widening of a few cortical sulci. She died of pneumonia at the age of 29 and a necropsy was not performed.

\section{Discussion}

There has been recent interest in congenital muscular dystrophies associated with involvement of the CNS, both of white matter and the cerebral cortex. As with muscular dystrophies in general, the field is expanding rapidly both on the genetic front and in terms of understanding the molecular genetic basis of these disorders and the cytoskeletal structural abnormalities that accompany the gene defects.

The membrane organisation of the dystrophin glycoprotein complex which provides linkage between the extracellular matrix and cytoskeleton has been reviewed. ${ }^{910}$ A dystrophin associated protein complex (including adhalin) binds intracellularly to dystrophin and extracellularly to merosin (a-2-laminin). The role of abnormal or absent dystrophin in the Xp21 muscular dystrophies is well known. ${ }^{1}$ Merosin deficiency is found in some of the occidental cases of congenital muscular dystrophy. ${ }^{11}$ Adhalin (both primary and secondary deficiency) has been implicated in the autosomal recessive severe childhood onset muscular dystrophy. A defect of $a$-sarcoglycan is also reported in which adhalin is deficient. ${ }^{10} 12$ Deficiency of one of the four sarcoglycan proteins leads to a reduction in the others, because of their interdependence for stability.

The different involvement of the CNS between the Japanese Fukuyama-type congenital muscular dystrophy and some of the cases described in the west has been noted for some time although the relevance of these findings was uncertain. ${ }^{5}$ In Fukuyama-type congenital muscular dystrophy, the major cerebral lesions and mental subnormality were consistent with disorders of neuronal migration, and although hypodensity of white matter was also noted this could disappear with age, suggesting delayed myelination. In the occidental (classic, pure) form, on the other hand, white matter translucencies on $\mathrm{CT}$ were the main $\mathrm{CNS}$ finding and were compatible with normal intelligence.

Some of the occidental cases can now be separated out as merosin deficient on immunocytochemical staining. ${ }^{11}$ In two series of biopsies from classic congenital muscular dystrophies quoted by Dubowitz, ${ }^{1}$ merosin was found to be absent in $45 \%$ and $65 \%$. Patients with merosin deficient congenital muscular dystrophy have associated white matter abnormalities on MRI, abnormal evoked potentials, and reduced motor nerve 
conduction velocities suggesting the presence of myelin abnormalities in the CNS and peripheral nervous system. ${ }^{12}$ In such cases linkage has been established to chromosome $6 \mathrm{q}$ near the merosin gene $^{9}$ and a mutation found. ${ }^{\text {i }}$

The specific abnormality in Fukuyama-type congenital muscular dystrophy remains unknown, although dystrophin associated proteins have low expression. ${ }^{8}$ This condition is autosomal recessive and, as already stated, is associated with developmental CNS abnormalities. Stable, often severe, mental retardation is invariable and febrile convulsions, epilepsy, and an abnormal EEG are common. These clinical features reflect cortical dysgenesis with abnormalities of the cerebral gyrae, including pachygyria, the most common CNS anomalies found. Other abnormalities may occur, including ventricular dilatation or focal interhemispheric fusion. A recent report suggested that three dimensional brain surface MRI may be useful diagnostically in suspected Fukuyama-type congenital muscular dystrophy. ${ }^{14}$

In patient 1 , merosin, adhalin, and dystrophin I and III were normal on muscle biopsy with only dystrophin II staining showing a few fibres with patchy negative areas. Histological findings were characteristic of a longstanding dystrophic process. On clinical grounds our two cases overlap with Fukuyama-type congenital muscular dystrophy, and the high creatine kinase concentration in patient 1 at the age of 21 is consistent. However, both our patients showed atypical features-namely, limited facial involvement, retained ambulation, severe retrocollis, and contractures. Brain MRI in patient 1 did not disclose any abnormalities of cortical gyrae despite volumetrically acquired scans and three dimensional reconstruction although ventricular dilatation was noted in both patients.

The severe retrocollis is of particular interest and is not a recognised feature in muscular dystrophies. Continuous activity in paraspinal muscles was found as shown on EMG and by persistent neck extension during sleep. This suggests that the abnormal posture does not simply reflect an imbalance of muscle power between neck extensors and flexors, and implies a dystonic component. The retrocollis of patient 1 is being cautiously treated with botulinum toxin injections with the aim of improving function and ambulation by achieving forward vision.

Whether these two siblings represent less severe forms of Fukuyama-type congenital muscular dystrophy is uncertain. The full range of this disease will only be known when the gene or specific cytoskeletal abnormality is identified. It may then be possible to begin to consider other, non-Fukuyama-type, merosin positive, congenital muscular dystrophies.

We thank Dr S Sisodiya, Epilepsy Research Group, Institute of Neurology, for the three dimensional volumetric MRI and Professor C D Marsden for referring the patient. We also thank Mrs Peachey and Dr J A Morgan-Hughes for the muscle biopsy.

1 Dubowitz V. The muscular dystrophies. In: Dubowitz V, ed. Muscle disorders of childhood. 2nd ed. London: WB Saunders 1995:34-133.

2 Toda $T$, Yoshioka $M$, Nakahori Y, Kanazawa I, Nakamura Y, Nakagome Y. Genetic identity of Fukuyama-type congenital muscular dystrophy and Walker-Warburg syndrome. Ann Neurol 1995;37:99-101.

3 Kimura S, Sasaki Y, Kobayashi T, Ohtsuki N, Tanaka Y, Hara M, et al. Fukuyama-type congenital muscular dystrophy and the Walker-Warburg syndrome. Brain Dev trophy and the

4 Toda T, Segawa M, Nomura Y, Nonaka I, Masuda K, Ishihara $\mathrm{T}$, et al. Localization of a gene for Fukuyamatype congenital muscular dystrophy to chromosome 9q31-33. Nature Genet 1993;5:283-6.

5 Gordon N. Muscle and brain disease: an update. Child Care Health Dev 1994;20:279-87.

6 Yoshioka M, Kuroki S, Nigami H, Kawai T, Nakamura H. Clinical variations within sibships in Fukuyama-type congenital muscular dystrophy. Brain Dev 1992;14:334-7.

7 Ranta S, Pihko H, Santavuori P, Tahvanainen E, De La Chappelle A. Muscle-eye-brain disease and Fukuyama type congenital muscular dystrophy are not allelic. Neuromusc Disord 1995;5:221-5.

8 Matsumura K, Nonaka I, Campbell K. Abnormal expression of dystrophin-associated proteins in Fukuyama-type congenital muscular dystrophy. Lancet 1993;341:521-2.

9 Campbell $\mathrm{K}$. Three muscular dystrophies: loss of cytoskeleton-extracellular matrix linkage. Cell 1995;80:675-9.

10 Brown R. Editorial review. Dystrophin-associated proteins and the muscular dystrophies: a glossary. Brain Pathol 1996;6:19-24.

11 Tom F, Evangelistica T, Leclerc A, Sunada Y, Manole E, Estournet B, et al. Congenital muscular dystrophy with merosin deficiency. CR Acad Sci 1994;317:351-73.

12 Dubowitz V, Mercuri E, Muntoni F, Philpot J, Sewry CA, Shorer Z. Peripheral and central nervous system involvement in merosin-deficient congenital muscular dystrophy. Neurology 1995;45(suppl 4):A407.

13 Bönnemann CG, Modi R, Noguchi S, Mizuno Y, Yoshida $M$, Gussoni E, et al. $\beta$-sarcoglycan (A3b) mutations $\mathrm{M}$, Gussoni $\mathrm{E}$, et al. $\beta$-sarcoglycan (A3b) mutations cause autosomal recessive muscular dystrophy with loss

14 Toda T, Watanabe T, Matsumura K, Sunada Y, Yamada T, Nakano I, et al. Three-dimensional MR imaging of brain surface anomalies in Fukuyama-type congenital muscular dystrophy. Muscle Nerve 1995;18:508-17. 